\title{
THEORETICAL STUDIES OF HADRONS AND NUCLEI
}

Final Progress Report

June 1997 - July 2006

\author{
Stephen R. Cotanch \\ Department of Physics \\ North Carolina State University \\ Raleigh, North Carolina 27695-8202
}

PREPARED FOR THE U. S. DEPARTMENT OF ENERGY UNDER GRANT NUMBER DE-FG02-97ER41048 


\section{INTRODUCTION}

This report details final research results obtained during the 9 year period from June 1, 1997 through July 15, 2006. The research project, entitled "Theoretical Studies of Hadrons and Nuclei", was supported by grant DE-FG02-97ER41048 between North Carolina State University [NCSU] and the U. S. Department of Energy [DOE]. In compliance with grant requirements the Principal Investigator [PI], Professor Stephen R. Cotanch, conducted a theoretical research program investigating hadrons and nuclei and devoted to this program $50 \%$ of his time during the academic year and $100 \%$ of his time in the summer. Highlights of new, significant research results are briefly summarized in the following three sections corresponding to the respective sub-programs of this project. Recent progress is also discussed in a recent renewal/supplemental grant proposal submitted to DOE. Finally, full detailed descriptions of completed work can be found in the publications listed at the end of this report.

\section{A. Hadron Structure}

\section{Many-Body Effective Hamiltonian Approach}

The focus of this project is the study of the the quark and gluon structure of hadrons, especially exotic systems (non $q q q$ or $q \bar{q}$ components). The long term goal is to obtain a complete description of hadrons from fundamental Quantum Chromodynamics [QCD] and to obtain a dynamic understanding of color confinement. Before detailing significant results it is appropriate to provide a brief synopsis of this project and related sub-projects.

This program approaches hadron structure from a nuclear physics many-body perspective and approximately diagonalizes an effective QCD Hamiltonian. There are two sub-programs; 1) obtain from the exact $\mathrm{QCH}$ Hamiltonian, $H$, an effective Hamiltonian, $H_{\text {eff }}$, that describes color confinement; 2) use many-body techniques and large-scale computations to diagonalize $H_{\text {eff }}$ for the hadron states including the ground state (QCD vacuum). We have specifically chosen the Coulomb gauge since it is amendable to a Hamiltonian formulation and also is rigorously renormalizable in this gauge. Further, and as detailed by Zwanziger, formulation in this gauge is free from the Gribov problem. A few years ago, using the similarity transformation technique of Glazek and Wilson, we were able to renormalize our Hamiltonian perturbatively to remove sensitivity to the regularization (truncation) schemes that were necessary to obtain a calculable Hamiltonian [4, 7]. We also applied flow equations, which are a series of continuous similarity transformations, to obtain a $H_{\text {eff }}[13,15]$. We initially used a linear confining potential with slope predetermined from lattice simulations for the energy of heavy static quarks as a function of distance. More recently, we have used an improved, self-consistent effective confining interaction. For a given $H_{\text {eff }}$ we then use many-body techniques to approximately diagonalize for the ground 
state (QCD model vacuum) and hadron states having quantum numbers $J^{P C}$. For the ground state vacuum we employed the Bardeen, Cooper and Schreiffer [BCS] variational approach in which the free (current) quarks and (zero mass) gluons are transformed into dressed quasiparticles by first performing a similarity transformation (BCS rotation) of the Fock operators and then finding the rotation (gap) angle by minimizing the ground state energy with respect to this variational parameter. This yields the quark and gluon gap equations (formally equivalent to the Dyson-Schwinger propagator equation in the rainbow approximation). The quasiparticles acquire constituent masses, roughly 100 and $250 \mathrm{MeV}$ for the u/d and s quarks, respectively, and 800 $\mathrm{MeV}$ for the dressed gluon. Further, the respective quark and gluon condensates (Cooper pairs) are $-(100 \mathrm{MeV})^{3}$ and $(433 \mathrm{MeV})^{4}$. With this quasiparticle basis $H_{\text {eff }}$ is then diagonalized for the hadron states using the Tamm-Dancoff approximation [TDA] truncated at the 1 parton, 1 antiparton level [26]. For comparison we also diagonalized using the random phase approximation [RPA] because it conserves chiral symmetry, unlike the TDA [11]. The RPA is formally equivalent to the Bethe-Salpeter equation using an instantaneous kernel. Strengths of this approach are that $H_{\text {eff }}$ has only a few ( 1 or 2$)$ dynamical parameters, in contrast to typical quark models, and contains the same symmetries and parton (quark, gluon) field structure as $H$. Equally significant, it provides a unifying framework for both quarks and gluons with controllable approximations. Our first application reproduced the lattice glueball spectra and subsequently we correctly predicted the observed meson spectra as well as glueball and hybrid meson (a three-body quark, antiquark and constituent gluon) masses in good agreement with lattice gauge calculations.

\section{Application in the Gluon Sector}

We, with F. Llanes-Estrada (Pl's former student now tenured at the Univ. Complutense in Madrid) and P. Bicudro (Instituto Superior Tecnico in Lisbon), obtained significant new results in our application of $H_{\text {eff }}$ to the gluon sector to study glueballs. As detailed further in our key publication [48], we performed a large scale BCS/TDA variational relativistic three-body calculation for the $\mid g g g>$ glueball spectrum. We specifically wished to investigate the odd $J^{--}$ states (oddballs) to determine if there were Regge trajectories, document the odderon, and also determine the intercept. Table I lists our predicted masses and compares to other approaches, especially lattice.

To provide insight and document theoretical model uncertainty, we also calculated the same glueball states with a simpler nonrelativistic constituent gluon potential model having several model parameters. Using Jacobi coordinates and a large harmonic oscillator expansion, we exactly diagonalized this simpler Hamiltonian. The model parameters were taken from previous quark studies (used the $q \bar{q}$ funnel potential) and, only adjusting the spin-spin potential strength to optimize comparison with lattice $J^{++}$states, obtained the even signature Regge trajectory, $\alpha_{P}^{M}(t)=0.23 t+1.0$, which is consistent with the pomeron and also our trajectory using the 
TABLE I: Glueball quantum numbers and masses in MeV.

\begin{tabular}{|c|c|cccccc|}
\hline \hline Model & $J^{P C}$ & $0^{-+}$ & $1^{--}$ & $2^{--}$ & $3^{--}$ & $5^{--}$ & $7^{--}$ \\
\hline our work & color & $f$ & $d$ & $d$ & $d$ & $d$ & $d$ \\
& $S$ & 0 & 1 & 2 & 3 & 3 & 3 \\
$H_{\text {eff }}$ & $L$ & 0 & 0 & 0 & 0 & 2 & 4 \\
$H_{M}$ & & 3900 & 3950 & 4150 & 4150 & 5050 & 5900 \\
lattice (Morningstar/Peardon) & & 3400 & 3490 & 3660 & 3920 & 5150 & 6140 \\
lattice (Meyer/Teper) & & 3640 & 3850 & 3930 & 4130 & & \\
Wilson loops (Simonov) & & 3250 & 3100 & 3550 & 4150 & & \\
\hline \hline
\end{tabular}

more sophisticated $H_{\text {eff }}$ approach. The $\mid g g g>$ glueball states predicted by this model $\left(H_{M}\right)$ are also listed in the table. The difference between approaches is an overall indication of theoretical uncertainty which is at the 10 to $15 \%$ level. Both of our model calculations yielded an odderon, $\alpha_{O}^{\text {eff }}(t)=0.23 t-0.880$ and $\alpha_{O}^{M}(t)=0.18 t+0.25$, having slopes similar to the pomeron but much lower intercepts, even below the $\omega$ meson intercept value of 0.5 . All results are pictorially summarized in Fig. 1. This is one of our important findings and indicates that it will not be possible to observe the odderon in high energy scattering processes in which the pomeron contributes since the cross section scales as $s^{1-\alpha(0)}$ and is dominated by the trajectory with the largest intercept. It may be possible to still observe the odderon in reactions having $C$ parity $=$ - 1 exchange where the pomeron is not present.

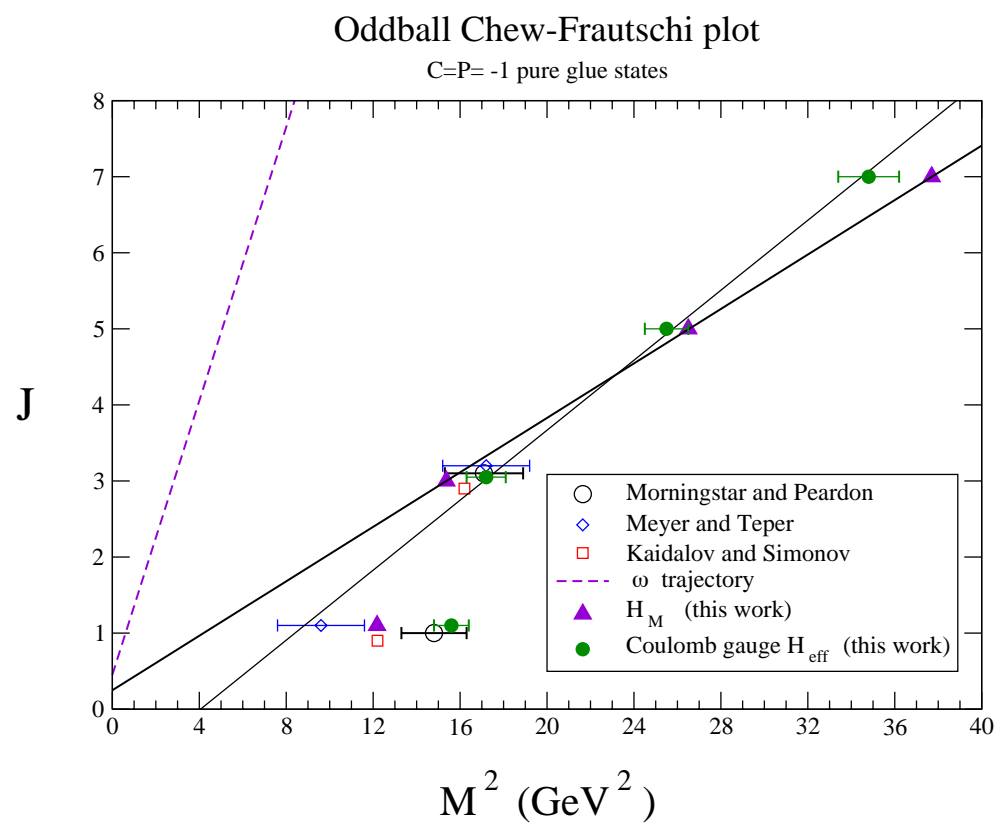

FIG. 1: Odderon trajectory from three-gluon spectroscopy and lattice compared to the $\omega$ meson Regge trajectory. 


\section{Application in the Quark Sector}

In our project involving $H_{\text {eff }}$ applications to the quark sector, several papers $[11,26,39,40$, 42] were published detailing significant, new findings. We have utilized a confining interaction that was determined self-consistently from our model QCD vacuum and also included a hyperfine interaction that was an effective vector-vector potential with generic form following from QCD. The hyperfine interaction significantly increased both the quark constituent mass and condensate yielding improved results for each and, for the first time, simultaneously described the small, $\approx$ $100 \mathrm{MeV}, J / \psi$ spin splitting and very large $\pi-\rho$ mass difference with a simple interaction. Table II summarizes our predictions for the light and heavy pseudoscalar and vector meson spectra. We have also predicted the vector meson leptonic decay widths, $\Gamma_{V \rightarrow e^{+} e^{-}}$, using the same $H_{\mathrm{eff}}$ as shown in the table. The predicted masses are in reasonable agreement especially since no fit was attempted (dynamic parameters and quark masses were not fine-tuned). For comparison, two other theoretical calculations are also presented. It is noteworthy that our few parameter approach provides a description which is comparable to these multi-parameter models.

In typical quark model formulations, a complicated multi-parameter hyperfine interaction is used to reproduce the the $\pi$ - $\rho$ mass differ, however applying the same interaction to excited $\pi$ - $\rho$ states, as well as to $J / \psi$ spin splittings, produces unphysically large mass differences. This is because chiral symmetry is generally not incorporated which our RPA approach does (produces a small pion mass, Goldstone boson). By comparing with our TDA $\pi-\rho$ result, which does not

TABLE II: Observed and predicted vector meson masses and widths compared to other calculations marked ${ }^{1}$ (Godfrey and Isgur $S$ - $D$ wave results without mixing) and ${ }^{2}$ (Ebert, Faustov and Galkin).

\begin{tabular}{c|cc|ccc} 
State & Exp. $M(\mathrm{MeV})$ & Calculated & Exp. $\Gamma_{e^{-} e^{+}}(\mathrm{keV})$ & Calculated & Other works \\
\hline$\rho$ & 770 & 795 & 6.85 & 4.7 & $6.87^{1}$ \\
$\omega$ & 782 & 795 & $0.60(2)$ & 0.50 & $0.85^{1}$ \\
$\phi$ & 1019 & 1005 & $1.26(2)$ & 1.1 & $2.75^{1}$ \\
$\omega$ & 1420 & 1420 & $0.1(1)^{\dagger}$ & 0.18 & $0.046^{1}$ \\
$\rho$ & $1450(25)$ & 1420 & - & 1.5 & $0.45^{1}$ \\
$\omega$ & $1650(25)$ & 1620 & - & 0.23 & $0.013^{1}$ \\
$\phi$ & $1680(25)$ & 1670 & $0.5(10)^{\dagger}$ & 0.21 & $0.27^{1}$ \\
$\rho$ & 1700 & 1520 & $3.5(5)^{\dagger}$ & 2.0 & $0.14^{1}$ \\
$\phi$ & - & 1790 & - & $0.45-0.55$ & $0.06^{1}$ \\
$J / \psi$ & 3097 & 3130 & $5.2(1)$ & 3.8 & $9.9^{1}, 5.4^{2}$ \\
$\psi$ & 3686 & 3681 & $2.2(2)$ & 2.0 & $3.3^{1}, 2.4^{2}$ \\
$\psi$ & 3770 & 3695 & $0.26(4)$ & 0.68 & $0.10^{1}$ \\
$\psi$ & 4040 & 4140 & $0.75(15)$ & 1.7 & $1.8^{1}$ \\
$\psi$ & $4160(20)$ & 4150 & $0.77(23)$ & 0.56 & \\
$\psi$ & $4415(6)$ & 4535 & $0.47(10)$ & 0.26 & \\
$\Upsilon$ & 9460 & 9460 & $1.32(5)$ & 0.45 & $1.4^{1}, 1.3^{2}$ \\
$\Upsilon$ & 10023 & 9870 & $0.52(3)$ & 0.35 & $0.65^{1}, 0.5^{2}$ \\
$\Upsilon$ & 10365 & 9921 & $0.5(1)^{\ddagger}$ & 0.0003 & $0.45^{1}$ \\
$\Upsilon$ & 10580 & 10190 & $0.32(3)^{\S}$ & 0.29 & $0.34^{1}$ \\
\hline
\end{tabular}

$\dagger^{\dagger}$ our estimate ${ }^{\ddagger} \mu^{+} \mu^{-}$width (roughly equal) $\S$ recently reported by BaBar 
preserve chiral symmetry, we determined that the hyperfine interaction is only responsible for about $30 \%$ of the large $610 \mathrm{MeV} \pi$ - $\rho$ mass difference. Thus, chiral symmetry dominates and is responsible for the remaining difference. In addition to the hyperfine interaction we have also included $\mathrm{D}$ orbital waves in the $\rho$ wavefunction which leads to coupled equations with $\mathrm{S}$ waves since our relativistic formulation naturally generates a tensor interaction. For the TDA there are two coupled equations while for the RPA, which already entails coupled $X$ and $Y$ functions, there are now four. The resulting equations are also nonlocal and are solved in momentum space. The D wave effect is not large but does slightly improve the meson description.

Other research highlights were calculating the $J$ reliability parameter used in lattice gauge simulations and predicting the mass of the $\eta_{b}$, which is still unconfirmed, to be about $9400 \mathrm{MeV}$ which will assist $B$ factory searches. Finally we also calculated the mass for the only missing vector meson below $2 \mathrm{GeV}, \phi(1880)$, to be $1790 \mathrm{MeV}$ consistent with multi-parameter quark models. Because this is the only missing hadron below $2 \mathrm{GeV}$ with $J^{P C}=1^{--}$, our specific signature results will aid searches for ortho baryonium (same quantum numbers) in this mass region which have been motivated by the para $(\mathrm{J}=0)$ baryonium interpretation of recent BES narrow resonance data near the $e^{-} e^{+} \rightarrow p \bar{p}$ threshold.

\section{Application in the Combined Quark and Gluon Sector}

There has also been progress in the combined quark and gluon sector project investigating hybrid mesons $\mid q \bar{q} g>$. These are nonconventional hadrons that can have exotic quantum numbers not possible in $q \bar{q}$ systems. With I. General (PI's graduate student supported by the grant) we have included the hyperfine interaction, discussed above, in our existing code that previously calculated the lightest exotic $1^{-+}$hybrid to have mass above $2 \mathrm{GeV}$. Our motivation is to determine if the hyperfine interaction will alter that result since the two observed $1^{-+}$states by the E852 collaboration were reported to have masses at 1.4 and $1.6 \mathrm{GeV}$ and were considered hybrid candidates. Our previous published hybrid calculation [14, 17], which agreed with both lattice and flux tube models, predicted a mass near but above $2 \mathrm{GeV}$ and suggested the observed states were more likely 4 quark states $\mid q \bar{q} q \bar{q}>$ which also can have exotic quantum numbers. We have finished a computationally intensive Monte Carlo calculation and published [50,51] results summarized below. This is part of Gerneral's PhD dissertation which he has completed and has now graduated (currently a postdoc at University of Pittsburgh).

We extended our previous hybrid study $[14,17]$ by including a new Hamiltonian term containing the $\mathbf{J}^{a} \cdot \mathbf{A}^{a}$ operators. Following our quark paper [39], an effective quark hyperfine interaction with a $\mathbf{J}^{a} \cdot \mathbf{J}^{a}$ form is obtained using perturbation theory to second order in $g$ and integrating over the gluonic degrees of freedom. This contribution to the hybrid mass is represented by the $q \bar{q}$ gluon exchange Feynman diagrams in Fig. ?? (first two in the bottom row). The non-abelian magnetic field terms are also included which entail triple-gluon vertices (last two diagrams). The remaining 


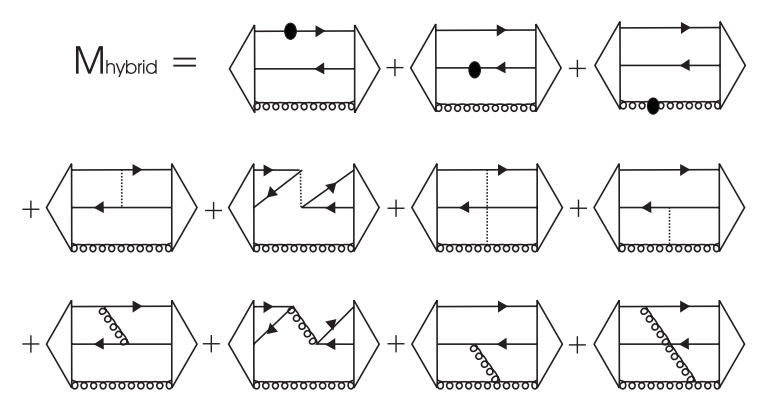

FIG. 2: Hybrid meson diagrams for $\left\langle\Psi_{q \bar{q} g}^{J P C}\left|H_{\text {eff }}\right| \Psi_{q \bar{q} g}^{J P C}\right\rangle$.

diagrams represent the self-energy, scattering and quark annihilation mass contributions.

The hybrid meson mass was computed variationally (see Ref. 51). The 9 and 12 dimensional integrals were calculated using the Monte Carlo method and repetitively evaluated with an increasing number of points until a weight-averaged result converged, typically involving about 50 million samples. The hybrid mass error introduced by this procedure is about $\pm 50 \mathrm{MeV}$. For each $J^{P C}$ hybrid state we optimized the two variational parameters.

Using standard current quark masses, $m_{u}=m_{d}=5 \mathrm{MeV}, m_{s}=80 \mathrm{MeV}$ and $m_{c}=1000$ $\mathrm{MeV}$, the predicted low-lying mass spectra for light and heavy hybrid mesons are presented in Figs. ?? and ??, respectively. Note that quark annihilation interactions increase the hybrid mass and this introduces isospin splitting since it only contributes in the $I_{q \bar{q}}$ channel. More importantly, all hybrid masses, especially the lightest exotic $1^{-+}$state, are clearly above $2 \mathrm{GeV}$. This is consistent with lattice and Flux Tube model results. These composite predictions strongly suggest that observed $1^{-+} \pi(1600)$, and more clearly $\pi(1400)$, are not hybrid meson states.

The charmed $c \bar{c} g$ hybrid spectrum has a slightly different level order compared to the the strange $s \bar{s} g$ and $u \bar{u} g$ spectra due to the hyperfine interaction. The predicted strange and charmed exotic $1^{-+}$states are also in reasonable agreement with both lattice and Flux Tube results.
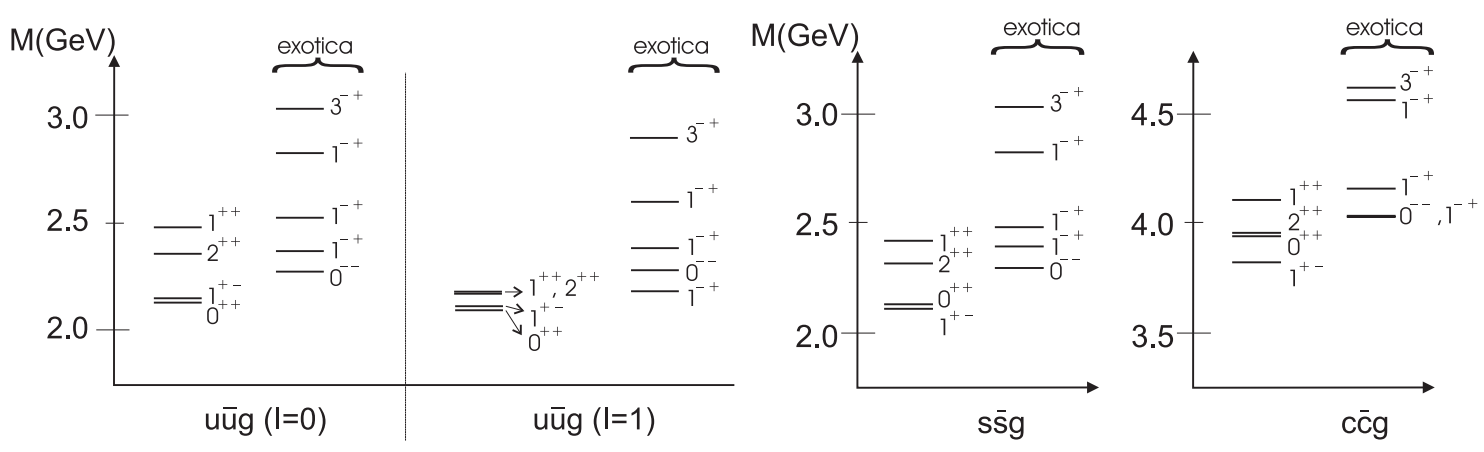

FIG. 3: Low lying $u \bar{u} g, s \bar{s} g$ and $c \bar{c} g$ spectra. 


\section{B. Probing Hadrons and Hadron Systems Electromagnetically}

This more phenomenological sub-program combines elements from QCD, QHD [Quantum Hadrodynamics], Regge theory and vector meson dominance [VMD] to analyze electromagnetic processes measured at GeV electron facilities, especially JLab. The goals are multifaceted and include searching for exotic hadron states (glueball photoproduction and decay), the nucleon strangeness content and meson electromagnetic production.

\section{Glueball Photoproduction and Decay}

New results have been obtained in this project with R. Williams (former PI student) which focuses on the important issue of observing glueballs. Two key papers have been published $[44,46]$ documenting the feasibility of detecting glueballs using the photoproduction process $p(\gamma, G) p$. The first paper [44] focused upon scalar, $0^{++}$, glueball $\left(G_{0}\right)$ photoproduction and decay and utilized the glueball-pomeron hypothesis to obtain the glueball hadronic coupling constant. The second paper [46] addressed tensor, $2^{++}$, glueball $\left(G_{2}\right)$ photoproduction and decay and reported a potentially useful decay profile for identifying glueballs. Using VMD and the flavor independence of gluon-quark coupling, the hadronic $G \rightarrow V V^{\prime}$ and radiative $G \rightarrow V \gamma$ widths were predicted for the vector mesons $V=\rho, \omega$ and $\phi$. The hadronic $V V^{\prime}$ decays are comparable for different vector meson channels with the $\omega \phi$ width the largest. However, the radiative $\omega \gamma$ and $\phi \gamma$ decays are suppressed relative to $\rho \gamma$ by over a factor of 2 which is distinct from typical meson branching rates. This novel $\omega \phi \rightarrow 3 \pi K \bar{K}$ branch, in conjunction with suppressed $\omega \gamma, \phi \gamma$ transitions relative to $\rho \gamma$, may be a useful glueball detection signature.

The tensor and scalar photoproduction cross sections are displayed in Fig. 5 which depicts the lab energy dependence for the forward $\mathrm{cm}$ angle $\theta_{\mathrm{cm}}=0^{\circ}$. While the scalar glueball cross section is somewhat larger, it is significant that the magnitude of both cross sections is sufficient to expect reasonable count rates. Indeed, measurements of this process, including vector meson decays, would appear feasible for the envisioned Hall D project at Jefferson Lab.

The photon transverse asymmetry observables, $A_{\gamma \perp}$, were also calculated and are displayed in Fig. 5 versus the $\mathrm{cm}$ angle. The scalar glueball asymmetry is greater than the tensor and both are large and increase with energy. This work relates to JLab activities, especially the CEBAF energy upgrade and Hall D spectrometer.

\section{Compton Scattering and Nucleon Strangeness}

This project investigates both time-like [TVCS] and space-like virtual Compton scattering [SVCS]. Each is separately described. 

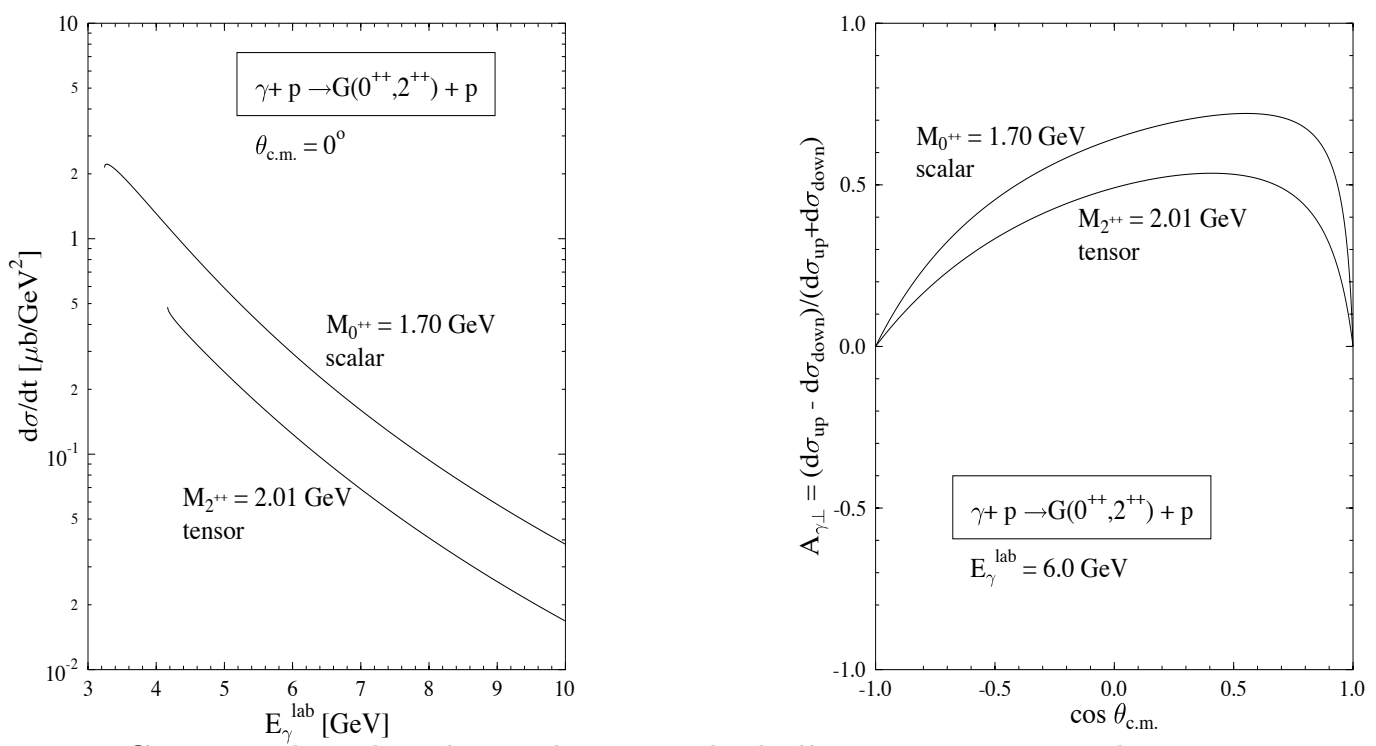

FIG. 4: Predicted scalar and tensor glueball cross sections and asymmetries.

The TVCS study focuses on the nucleon strangeness using the reaction, $N\left(\gamma, e^{+} e^{-}\right) N$. We have previously demonstrated through QHD calculations and extended VMD that measurements of this process will provide new information about the nucleon electromagnetic form factors in the still unmeasured $q^{2} \leq 4 M_{N}^{2}$ time-like region which is kinematically inaccessible using two-body final state reactions. In several publications [1, 5, 24], we predicted dramatic, several-order of magnitude narrow resonances from vector meson contributions. Including $\phi N N$ coupling in the nucleon form factor yields a unique dual peak experimental cross section signature for assessing possible OZI suppression or evasion and the related strangeness content of the nucleon. We developed a high $t$ theorem asserting that cross section ratios for the resonant peaks corresponding to $\phi$ and $\omega$ scales as the ratio of coupling constants squared, $g_{\phi}^{2} / g_{\omega}^{2}$. Our predictions provide a novel signature for measuring OZI violations and the related strangeness content of the nucleon since the coupling $g_{\phi N N}^{V}$ is directly related to the nucleon vector current matrix element, $<N\left|\bar{s} \gamma_{\mu} s\right| N>$. Because the photon has the same quantum numbers as the $\phi$ meson we have used the same QHD formalism that successfully describes recently measured $\phi$ photoproduction data. Both of these processes entail $t$ channel pomeron exchange and this relates to our glueball studies.

We also published a paper [45] documenting the relative importance of the intermediate two photon Bethe-Heitler [BH] process, $\gamma p \rightarrow p+\gamma+\gamma^{*} \rightarrow e^{+} e^{-}+p$, which can compete with the one photon time-like virtual Compton process $\gamma p \rightarrow p+\gamma^{*} \rightarrow e^{+} e^{-}+p$. In the $\mathrm{BH}$ process the two photons are in a positive $C$ parity state producing 2 leptons also having $C=1$. This is in 
contrast to TVCS involving a single photon and a negative $\mathrm{C}$ parity $e^{+} e^{-}$state. By calculating the $\mathrm{BH}$ amplitude and measuring the lepton charge asymmetry, $\sigma\left(e^{+} e^{-}\right)-\sigma\left(e^{-} e^{+}\right)$, one can experimentally extract the TVCS amplitude since the interference term is odd under $C$ parity or lepton interchange. We (with $\mathrm{PI}$ postdoc $\mathrm{C}$. Kao) calculated both processes and documented the appropriate kinematics to optimize this procedure. In general the TVCS process is comparable to the $\mathrm{BH}$ except for small $t$ or forward angles where the $\mathrm{BH}$ amplitude dominates. This work relates to JLab and we have been collaborating with $\mathrm{H}$. Gao (Duke) regarding future $\mathrm{Hall} \mathrm{B}$ and the envisioned Hall D measurements.

The SVCS investigation centers on using Heavy Baryon Chiral Perturbation Theory [HBChPT] to calculate the Compton amplitude and obtain the nucleon spin flip generalized polarizabiliites [GPs]. Kao (PI's postdoc) is an expert on HBChPT and he calculated the next-to-next leading order $\mathcal{O}\left(p^{4}\right)$ spin-dependent SVCS amplitude. At this order there are no unknown low-energy constants in the theory and the GPs can be predicted without ambiguity. This next order calculation revealed important corrections from previous, lower order results and the GPs are now closer in agreement to phenomenological GPs estimated from dispersion relations. This work also detailed how to extract the spin-flip GPs from both unpolarized SVCS measurements as well as by using double polarization observables and predictions were made for experimental conditions accessible at MAMI.

\section{Meson Electromagnetic Production}

This project has investigated kaon, pion and $\phi$ meson electromagnetic production and radiative capture for proton and nuclear targets. Results documented the novel physics associated with the strange quark systems of hyperons, few hyperon systems and hypernuclei. In particular, we assisted searches for possible exotic resonances, furthered the understanding of fundamental $s$ quark production and hyperon-hadron interactions, and studied hypernuclear structure.

The principle motivation for investigating elementary $K^{+}$electromagnetic production and the crossing related $K^{-}$radiative capture process is to understand the fundamental reaction mechanism at the quark level and the constituent structure of the participating hadrons. The difficulties with nonperturbative QCD still necessitates the utilization of QHD reaction calculations. Unless the coupling constants are small, simple QHD formulations will generally violate unitarity and are also not renormalizable. This approach enjoys, however, many phenomenological successes and embodies several important features including field theory, gauge and Lorentz invariance, duality (Regge theory) and crossing symmetry. Without additional improvements (some mentioned above), it is a continuing debate whether this approach will determine the hadronic coupling constants, however, it should be adequate for determining electromagnetic vertices and form factors which in turn can be calculated more easily from QCD. We also performed and completed more fundamental meson production calculations within the quark Dyson-Schwinger (DS) formalism 
detailed below.

We have applied our unified model $[3,5,8,9]$ for pseudo-scalar meson production and capture to the latest Hall C JLab data for $\mathrm{p}\left(\mathrm{e}, \mathrm{e} K^{+}\right) \Lambda$. The unprecedented high count rate for exclusive $\mathrm{p}\left(\mathrm{e}, \mathrm{e} K^{+}\right) \Lambda$ measurements has permitted extracting the most accurate transverse and longitudinal cross sections to date. Our predictions gave a good description of this data for the sum and separated transverse, $\sigma_{T}$, and longitudinal, $\sigma_{L}$, cross sections with no change in the QHD model parameters that have provided a good $\chi^{2}$ fit to the previous world data set.

Relating to elementary $\Lambda$ and $\Sigma$ production, a paper was published [37] concerning a new constraint relation for the $\Lambda$ and $\Sigma$ coupling constants using the generalized Goldberger-Treiman [GT] relation and the Dashen-Weinsten sum rule. This result provided a more stringent criteria for determining the poorly known hyperon coupling constants. We comprehensively examined all published hyperon couplings and applied the constraint to obtain an improved reduced subset consistent with this criteria which spans the intervals $0.80 \leq g_{K \Sigma N} / \sqrt{4 \pi} \leq 2.72$ and $-3.76 \leq$ $g_{K \Lambda N} / \sqrt{4 \pi} \leq-1.84$. The broken $S U_{F}(3)$, GT and extrapolated Taylor series couplings are also in this acceptable interval. This constraint will benefit experimentalist studying $K$ scattering and production at hadron and electron beam facilities.

With P. Maris (Pl's postdoc) we applied the DS approach to $\pi \pi$ scattering system and from a large-scale, supercomputer based calculation, documented that it is necessary to go beyond the impulse approximation and include all ladder diagrams (gluon exchanges) in the $\mathrm{s}$ and $\mathrm{t}$ channels to reproduce the known low energy amplitude behavior. In particular we numerically agreed with Weinbergs theorem for the different isospin scattering lengths and at higher energies reproduced the $\sigma$ and $\rho$ resonances. We also made a consistent comparison to QHD meson exchange models by using the DS calculated parameters (masses, widths, coupling constants) in the QHD amplitudes. We found good agreement and concluded that the QHD models were reasonable. This work was published $[22,25]$ and presented at several conferences.

We also extended this DS treatment to $\gamma \pi \rightarrow \pi \pi$ and were able to reproduce the fundamental low-energy theorem for the chiral anomaly. Extending our formalisms to the crossing related process $\gamma \rightarrow \pi \pi \pi$, we computed the anomalous form factor shown in Fig. 6 .

The asterisk are our full ladder DS calculation which is consistent with chiral symmetry and reproduces the low energy theorem at $s=0$ (chiral anomaly) and roughly agrees with the limited data which will soon be measured more accurately at Jlab. The other two curves are VMD (dotted) and a modified VMD (dashed) which reproduces the chiral anomaly. The phenomenological masses, widths and coupling constants are used in the VMD for most favorable prediction. If we use the calculated DS parameters in the VMD then both the full DS and VMD form factors agree to high accuracy. The DS is able to reproduce the underlying $\rho$ resonance structure and we concluded that the QHD approach, as in $\pi \pi$ scattering, is reasonable. This work was published in Ref. [33] and in a major invited review [31]. 


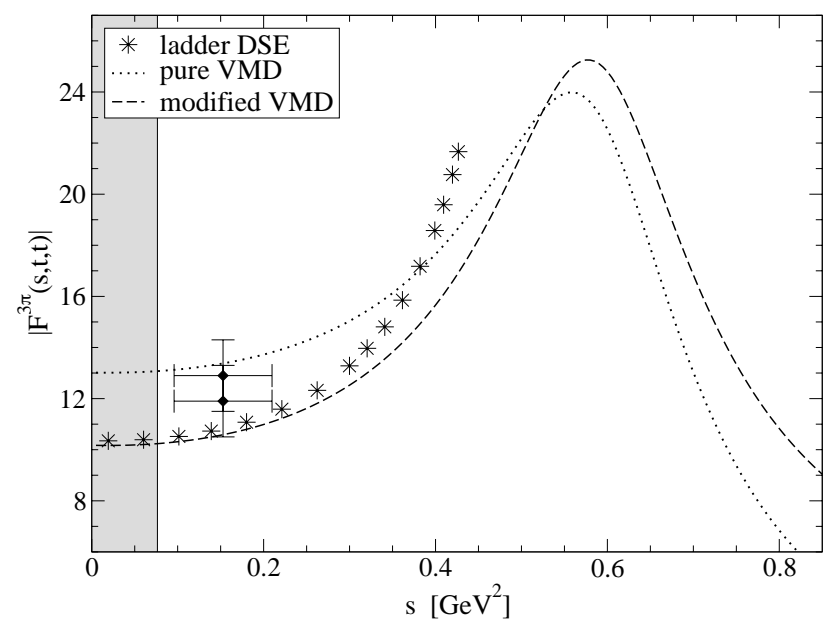

FIG. 5: Comparison of DSE (asterisk), pure VMD (dotted), and modified VMD (dashed) form factors, $F^{3 \pi}(s, t, t)$, with data at $Q^{2}=0$. The experimental coupling constants, masses and width are used in both VMD form factors (the shaded region is unphysical).

\section{Many-Body Studies}

The PI has an established history of activity in many-body physics, particularly for nuclear systems and has published many papers on the continuum shell model approach to nuclear relations, especially photonuclear.

\section{Continuum shell model approach to photonuclear reactions}

This long term project has utilized large-scale supercomputer based resources to investigate the structure and reaction mechanism for photonuclear reactions on light nuclei by performing continuum shell model calculations for $(\gamma, \mathrm{N})$ and $(\mathrm{p}, \gamma)$ reactions at low and medium energies spanning the $\Delta$ isobar region. The essence is a microscopic formulation in which a realistic many-body Hamiltonian $\mathrm{H}$ for the $\mathrm{A}$ baryon system is diagonalized in a large Banach space (not Hilbert since the continuum states are not square integrable) spanned by products of continuum single-particle states and self-consistent bound many-body eigenfunctions for the A-1 system. The Hamiltonian contains a realistic effective interaction with finite range, spin, isospin, tensor and $N-\Delta$ interaction components. Diagonalizing $\mathrm{H}$ in this space generates a large set of coupled inhomogeneous, integro-differential equations in two variables ( $\mathbf{r}$ and $\mathbf{r}^{\prime}$ due to non-localities) to be solved, subject to appropriate scattering boundary conditions, for the unknown partial-wave scattering functions. All non-localities are retained in the radial kernels due to wavefunction antisymmetrization, Pauli blocking and exchange. Delta isobar formation and propagation is explicitly included in the wavefunction by direct coupling to $\Delta$ channels through $N-\Delta$ inter- 
actions. By including a large number of scattering channels (up to 144) that rigorously account for loss of flux from the elastic channel, the optical potential is computed microscopically. Hence no phenomenological absorption is needed and $\mathrm{H}$ remains Hermitian assuring proper wavefunction orthogonality which is especially important in photonuclear reactions. Related, the code rigorously includes unitary and gauge-invariance. The electromagnetic interaction is expanded in multipoles and to achieve convergence for medium energy photons typically requires up to 20 electric and magnetic multipoles. For each energy and multipole we solve $\mathrm{N}$ coupled, non-local integro-differential equations which is equivalent to inverting a large supermatrix, with dimension of order 40,000 by 40,000 , requiring supercomputer computations. The model is detailed in our paper [6] and the PI has given an invited talk at a continuum shell model workshop in Trento $\left(E C T^{*}\right)$. Our code and formalism will be useful for envisioned studies at RIA especially for exotic nuclei that are weakly bound where continuum effects are important.

\section{BCS and Attractive Hubbard Model Comparative Study}

Related to our QCD hadronic studies using the BCS, this research assessed the accuracy of the BCS for a spin lattice system consisting of 1 and 2 dimensional electrons. A study was performed comparing the BCS approximation to exact diagonalizations of the attractive Hubbarb model $[\mathrm{AHM}]$ and a reduced model Hamiltonian which produced pairing and described $\mathrm{S}$ wave superconductivity. While not involving hadron physics, it is relevant since the methods, techniques and calculational tools are similar to those used in our hadronic structure project. In collaboration with N. Salwen, a NCSU postdoc supported by department funds, and PI graduate student S. Sheets the exact diagonalizations were compared to the BCS in both one and two dimensions. This was the first exact diagonalization of the AHM in two dimensions. The BCS was found to be an excellent approximation for energies and gaps for arbitrary strong potential energies. This provides further support for BCS applications to nonperturbative systems such as our model QCD vacuum described above. Our results also determined the AHM, which is known to not have long-range order in one dimension at zero temperature, does not exhibit a dimensional crossover, or density induced transitions, to long-range order in two dimensions which has been previously conjectured. This work was published in Ref. [36] and provided good training for Sheets who completed his Masters Degree research. Salwen is now a postdoc at Ohio State in the hadronic theory group.

\section{Many-Body Methods and Effective Field Theory}

More recently, a paper [47] was published using effective field theory [EFT] for a system of neutrons at moderate density appropriate to neutron stars. This, more formal, study was done in collaboration with T. Schaefer and C. Kao. In the framework of pionless nucleon-nucleon effective 
field theory, we studied different approximation schemes for the nuclear many body problem and evaluated ladder diagrams constructed from particle-particle, hole-hole, and particle-hole pairs. We focused on the problem of finding a suitable starting point for perturbative calculations near the unitary limit $\left(k_{F} a\right) \rightarrow \infty$ and $\left(k_{F} a\right) \rightarrow 0$, where $k_{F}$ is the Fermi momentum, $a$ is the scattering length and $r$ is the effective range. We also clarified the relationship between different classes of diagrams and the large $g$ and large $D$ approximations, where $g$ is the fermion degeneracy and $D$ is the number of space time dimensions. In the large $D$ limit we find that the energy per particle in the strongly interacting system is $1 / 2$ the result for free fermions.

\section{SERVICE AND NON-RESEARCH ACTIVITIES}

In addition to research, the investigators supported by the grant contributed to the nuclear community in service. In particular, they assisted in conferences and workshops and refereed numerous papers and proposals. More specfically, Cotanch was has served on several advisory and program committees with the most recent for the International Conference on Quark and Nuclear Physics (Madrid, June, 2006) and the 5th International Workshop on Chiral Dynamics (Durham, NC, September, 2006). He also has served on review panels and external advisory boards for physics departments in the US with the most recent being New Mexico State University and Florida International University which both have connections to JLab.

The investigators hosted visitors and speakers for the weekly Triangle Nuclear Theory (TNT) seminar program. The TNT seminar series, which is supported by DOE, has completed its sixteenth year and continues to be very successful. The stimulating talks by theorists not only provide an informative forum for educating triangle nuclear physicists but also help foster interactions between both theorists and experimentalists at NCSU, Duke and UNC. 


\section{PAPERS PUBLISHED IN THE REPORT PERIOD}

1. "Probing Nucleon Strangeness using Vector Meson Dominance", S. R. Cotanch and R. A. Williams, Nucl. Phys. A631, 478 (1998).

2. "QCD Hamiltonian Approach for the Glueball Spectrum", S. R. Cotanch, A. Szczepaniak, E. Swanson and C.-R. Ji, Nucl. Phys. A631, 640 (1998).

3. "Kaon Electromagnetic Production and $\phi$-Nucleon Coupling", S. R. Cotanch, Proceedings of the Physics of Strangeness Workshop, Vol. II, p. 1 (1998)

4. "Renormalized Effective QCD Hamiltonian: Gluonic Sector", D. G. Robertson, A. Szczepaniak, E. Swanson, C.-R. Ji and S. R. Cotanch, Phys. Rev. D 59, 074019 (1999).

5. "Electromagnetic Probe of Nucleon Strangeness", S. R. Cotanch and R. A. Williams, Few-Body Systems Suppl. (Springer-Verlag) 10, 419 (1999).

6. "Continuum Shell Model Calculations for Photonuclear Reactions", S. R. Cotanch, Proceedings of the Workshop on Electron Nucleus Scattering, Ed: O. Benhar, A. Fabroconi, R. Schiavilla, EDIZIONI ETS, 207-223 (1999).

7. "Renormalized Effective QCD Hamiltonian Approach", D. G. Robertson, A. Szczepaniak, E. Swanson, C.-R. Ji and S. R. Cotanch, Quark Confinement and the Hadron Spectrum III, eds. N. Brambilla, (World Scientific) (1999).

8. "Kaons and Glueballs at Jefferson Lab: a Strange, Sticky but Colorful Production", S. R. Cotanch, Hadron Physics 98, eds. E. Ferreira, F. F. de Souza Cruz and S. dos Santos Avancini (World Scientific), 236 (1999).

9. "Hyperon and Glueball Electromagnetic Production", S. R. Cotanch, Fizika B 8, No. 2, 387 (1999).

10. "Probing Nucleon Strangeness and QCD Many-Body Approach", S. R. Cotanch, Perspectives in Hadronic Physics, eds. S. Boffi, C. Ciofi degli Atti and M. Giannini (World Scientific), 255 (1999).

11. "Meson Structure in a Relativistic Many-Body Approach", F. J. Llanes-Estrada and S. R. Cotanch, Phys. Rev. Lett. 84, 1102 (2000).

12. "Many-Body QCD Approach to Hadrons", S. R. Cotanch, and F. J. Llanes-Estrada, LightCone QCD and Nonperturbative Hadron Physics, eds. A. Schreiber and A. G. Williams (World Scientific), 249 (2000). 
13. "Flow Equations for Gluodynamics in the Coulomb Gauge", E. Gubankova, C. Ji, and S. R. Cotanch, Phys. Rev. D 62, 074001 (2000).

14. "Coulomb Gauge Hybrid Meson Calculation", F. J. Llanes-Estrada and S. R. Cotanch, Nucl. Phys. B90, 117 (2000).

15. "Flow Equations for Quark-Gluon Interactions in Light Front QCD", E. Gubankova, C. Ji, and S. R. Cotanch, Phys. Rev. D 62, 125012 (2000).

16. "Relativistic Many-Body Approach to Exotic and Charmed Hybrid Mesons", S. R. Cotanch and F. J. Llanes-Estrada, Nucl. Phys. A689, 481 (2001).

17. "Many-Body Coulomb Gauge Exotic and Charmed Hybrids", F. J. Llanes-Estrada and S. R. Cotanch, Phys. Lett. B 504, 15 (2001).

18. "Mesons and Hybrids in a Relativistic Many Body Theory", S. R. Cotanch and F. J. Llanes-Estrada, AIP Conference Proceedings 549, 275 (2001).

19. "Charmonium, Glueballs and Exotic Hybrids in a Relativistic Many-Body Approach", S. R. Cotanch, Lepton Scattering, Hadrons and QCD, eds. A. Schreiber and A. G. Williams (World Scientific), 271 (2001).

20. "Hadrons in a Relativistic Many Body Approach", S. R. Cotanch and F. J. Llanes-Estrada, Hadrons, Nuclei and Applications (World Scientific), 209 (2001).

21. "Many-Body Approach to Mesons, Hybrids and Glueballs", S. R. Cotanch and F. J. LlanesEstrada, Quark Confinement and the Hadron Spectrum IV, ed. W. Lucha and K. M. Maung (World Scientific), 90 (2002).

22. "Chirally Symmetric Quark Description of Low Energy $\pi \pi$ Scattering", P. Bicudo, S. R. Cotanch, F. J. Llanes-Estrada, P. Maris, E. Ribeiro and A. Szczepaniak, Phys. Rev. D 65, 076008 (2002).

23. "QCD Glueball Regge Trajectory and the Pomeron", F. J. Llanes-Estrada, S. R. Cotanch, P. Bicudo, E. Ribeiro and A. Szczepaniak, Nucl. Phys. A710, 45 (2002).

24. "Probing Proton Strangeness with Time-Like Virtual Compton Scattering", S. R. Cotanch and R. A. Williams, Phys. Lett. B 549, 85 (2002).

25. "QCD Based Quark Description of $\pi \pi$ Scattering up to the $\sigma$ and $\rho$ Regions", S. R. Cotanch and P. Maris, Phys. Rev. D 66, 116010 (2002).

26. "Relativistic Many-Body Approach to Mesons", Felipe. J. Llanes-Estrada and Stephen R. Cotanch, Nuclear Physics A697, 303 (2002). 
27. "Proton Strangeness via Compton Scattering", S. R. Cotanch, Exclusive Processes at High Momentum Transfer (World Scientific), 294 (2002).

28. "Unified Many-Body Approach to Mesons, Glueballs and Hybrids", S. R. Cotanch, Prog. Part. Nucl. Phys. 50, 353 (2003).

29. "Facets of confinement and dynamical chiral symmetry breaking", P. Maris, A. Raya, C. D. Roberts and S. M. Schmidt, Eur. Phys. J. A 18, 231 (2003).

30. "Massless QED(3) with explicit fermions", D. Lee and P. Maris, Phys. Rev. D 67, 076002 (2003).

31. "Dyson-Schwinger equations: A tool for hadron physics", P. Maris and C. D. Roberts, Int. J. Mod. Phys. E 12, 297 (2003).

32. "Strong decays of light vector mesons", D. Jarecke, P. Maris and P. C. Tandy, Phys. Rev. C 67, 035202 (2003).

33. "Ladder Dyson-Schwinger Calculation of the Anomalous $\gamma$ - $3 \pi$ Form Factor", S. R. Cotanch and P. Maris, Phys. Rev. D 68, 036006 (2003).

34. "Glueball and Pomeron Daughter Trajectories", S. R. Cotanch, Nucl. Phys. A737, S15 (2004).

35. "Coulomb Gauge Hamiltonian: Advances and Applications", S. R. Cotanch, Fizika B 13, 27 (2004).

36. "BCS and Attractive Hubbard Model Comparative Study", N. Salwen, S. Sheets and S. R. Cotanch, Phys. Rev. B 70, 064511 (2004).

37. "Goldberger-Treiman Constrant Criteria for Hyperon Coupling Constants", I. General and S. R. Cotanch, Phys. Rev. C 69, 035202 (2004).

38. "Analytic properties of the Landau gauge gluon and quark propagators", R. Alkofer, W. Detmold, C. S. Fischer and P. Maris, Phys. Rev. D 70, 014014 (2004).

39. "Hyperfine qq- Splitting: Chiral Symmetry Versus Transverse Gluon Exchange", F. J. Llanes-Estrada, S. R. Cotanch, A. Szczepaniak and E. Swanson, Phys. Rev. C 70, 035202 (2004).

40. "Chiral Symmetry and Hyperfine qq- Splittings", F. J. Llanes-Estrada, S. R. Cotanch, A. Szczepaniak and E. Swanson, Eur. Phys. J C33, S521 (2004). 
41. "Higher moments of nucleon spin structure functions in heavy baryon chiral perturbation theory and in a resonance model", C. W. Kao, D. Drechsel, S. Kamalov and M. Vanderhaeghen, Phys. Rev. D 69, 056004 (2004).

42. "RPA Vector Meson Leptonic Widths", F. J. Llanes-Estrada and S. R. Cotanch, Proceedings of the "Encuentro de Fisica Fundamental Alberto Galindo" Meeting, Madrid Spain, nucl-th/0408038 (2004).

43. "New predictions for generalized spin polarizabilities from heavy baryon chiral perturbation theory, C. W. Kao, B. Pasquini and M. Vanderhaeghen, Phys. Rev. D 70, 114004 (2004).

44. "Glueball Enhancements in $\mathrm{p}(\gamma, \mathrm{VV}) \mathrm{p}$ through Vector Meson Dominance", S. R. Cotanch and R. A. Williams, Phys. Rev. C 70, 055201 (2004).

45. "Time-Like Compton Scattering and the Bethe-Heitler Process", S. R. Cotanch, Eur. Phys J. A24, 101 (2005).

46. "Tensor Glueball Photoproduction and Decay", S. R. Cotanch and R. A. Williams, Phys. Lett. B 621, 269 (2005).

47. "Many Body Methods and Effective Field Theory", T. Schafer, C.-W. Kao and S. R. Cotanch, Nucl. Phys. A762, 82 (2005).

48. "Oddballs and a Low Odderon Intercept", P. Bicudo, F. Llanes-Estrada and S. R. Cotanch, Phys. Rev. Lett. 96, 081601 (2006).

49. "Soft Gluons are Heavy and Rowdy", R. Alkofer, P. Bicudo, S. R. Cotanch, C. S. Fischer and F. J. Llanes-Estrada, Proceedings of the 2nd Workshop on Hadron Structure and QCD, St. Peterburg, Russia, in press (2006).

50. "QCD Coulomb Gauge Approach to Exotic Hadrons", S. R. Cotanch, I. General and P. Wang, Eur. Phys. J. A, in press (2007).

51. "QCD Coulomb Gauge Approach to Hybrid Mesons", I. General, S. R. Cotanch and F. Llanes-Estrada, Eur. Phys. J. C, in press (2007).

52. "The BES $f_{0}(1810)$ : a New Glueball Candidadate", P. Bicudo, F. Llanes-Estrada, S. R. Cotanch and D. G. Robertson, submitted to J. of Nuclear and Particle Physics G [hep$\mathrm{ph} / 0602172]$. 


\section{INVITED TALKS}

1. Kaons and Glueballs at Jefferson Lab: a Strange, Sticky but Colorful Way to do Physics, S. R. Cotanch, VI Hadron Physics 1998, Florianopolis, Brazil (1998).

2. Nucleon Strangeness Content Through Vector Meson Dominance, S. R. Cotanch, 16th European Conference on Few-Body Problems in Physics, Autrans, France (1998).

3. Continuum Shell Model Approach to Photonuclear Reactions, S. R. Cotanch, Workshop on Electron-Nucleus Scattering, Elba International Physics Center, Marciana Marina, Isola dElba, Italy (1998).

4. Kaon Electromagnetic Production and $\phi$-Nucleon Coupling, S. R. Cotanch, INT Workshop on the Physics of Strangeness, Seattle, Washington (1998).

5. Hyperon and Glueball Electromagnetic Production, S. R. Cotanch, International Conference on Nuclear and Particle Physics, Dubrovnik, Croatia (1998).

6. Probing Nuclear Forn Factors and the Nucleon Strangeness Content, S. R. Cotanch, 2nd International Conference on Perspectives in Hadronic Physics, ICTP, Trieste, Italy (1999).

7. Effective QCD Hamiltonian Approach to Hadrons, S. R. Cotanch, Gordon Research Conference on Dynamics of Simple Systems, Newport, RI (1999).

8. Many-Body QCD Hamiltonian Approach to Glueballs and Mesons, S. R. Cotanch, International Workshop Challenges in QCD, Kfar Giladi, Israel (1999).

9. Many-Body QCD Approach to Hadrons, S. R. Cotanch, Workshop on Light-Cone QCD and Nonperturbative Hadron Physics, Adelaide, Australia (1999).

10. Relativistic Many-Body Approach to Mesons, S. R. Cotanch, 7th Conference on the Intersections of Particle and Nuclear Physics, Quebec City, Canada (May, 2000).

11. Mesons and Hybrids in a Relativistic Many Body Theory, S. R. Cotanch, Bologna 2000, Structure of the Nucleus at the Dawn of the Century, Bologna, Italy, (June, 2000).

12. Many-Body Approach to Mesons, Hybrids and Glueballs, S. R. Cotanch, Quark Confinement and the Hadron Spectrum IV, Vienna, Austria (July, 2000).

13. Relativistic Many-Body Approach to Exotic and Charmed Hybrid Mesons, S. R. Cotanch, Europeon Few-Body Conference, Evora, Portugal (September, 2000).

14. Renormalized Effective QCD Hamiltonian, S. R. Cotanch, Workshop on Relativistic Dynamics and Few-Body Systems, Trento, Italy (November, 2000). 
15. Mesons, Glueballs and Exotic Hybrids in a Relativistic Many-Body Approach, S. R. Cotanch, Workshop on Lepton Scattering, Hadrons and QCD, Adelaide, Australia (April, 2001).

16. Many Body Approach to Hadron Structure, S. R. Cotanch, INT-01-1 Program on Correlations in Nucleons and Nuclei, Institute for Nuclear Theory, Seattle Washington (June, 2001).

17. Nucleon Strangeness via Time-Like Virtual Compton Scattering, S. R. Cotanch, Workshop on Exclusive Processes at High Momentum Transfer, Jefferson Lab, Newport New, VA (May, 2002).

18. Continuum Shell Model Approach to Photonuclear Reactions with Exchange Currents, Workshop on Continuum Aspects of the Nuclear Shell Model, ECT*, S. R. Cotanch, Trento, Italy (June, 2002).

19. Unified Relativistic Many-Body Description of Mesons, Hybrids and Glueballs, International School of Nuclear Physics: Quarks in Hadrons and Nuclei, Erice, Sicily (September, 2002).

20. Hadrons, Hyperons, Hybrids and Hamiltonians: a 4H Club at Jefferson Lab, S. R. Cotanch, 2nd International Conference on Nuclear and Particle Physics with CEBAF at Jefferson Lab, Dubrovnik, Croatia (May, 2003).

21. Glueballs and the Pomeron, S. R. Cotanch, 17th International Conference on Few-Body Problems in Physics, Durham, NC (June, 2003).

22. Explicit and Hidden Strangeness at Jlab: Selected Results and Some Ideas for the Future, S. R. Cotanch, Special Symposium on the Highlights of the First Seven Years at Jefferson Lab, Newport News, VA (June, 2003).

23. Compton Scattering and Photoproduction: A Quest for Nucleon Strangeness and Glueballs, S. R. Cotanch, Institute for Nuclear Theory Program on Generalized Parton Distrubutions and Hard Exclusive Processes (INT-03-2), Seattle, WA (July, 2003).

24. Nucleon Strangeness via $\mathrm{p}\left(\gamma, e^{+} e^{-}\right) \mathrm{p}$ and Glueball Excitation via $\mathrm{p}(\gamma, \mathrm{VV}) \mathrm{p}, \mathrm{S}$. R. Cotanch, III International Conference on Quarks and Nuclear Physics, Indiana University, Bloomington, IN (May, 2004).

25. Proton Strangeness with Time-like Virtual Compton Scattering, S. R. Cotanch, International Workshop on Parity Violation and Hadronic Structure, Laboratoire de Physique subatomique et de Cosmologie, Grenoble, France (June, 2004).

26. Oddball Regge Trajectory and a Low Odderon Intercept, S. R. Cotanch, Odderon Searches at RHIC Workshop, Brookhaven National Lab (Sept., 2005). 
27. QCD Coulomb Gauge Approach to Oddballs and Hybrid Mesons, S. R. Cotanch, IV International Conference on Quarks and Nuclear Physics, Madrid, Spain, (June, 2006).

Finally, the investigators presented several colloquia and seminars (nationally and internationally) as well as submitting contributed talks to meetings (references are available upon request). 